\title{
Energy and Spectral Efficiency Gains From Multi-User MIMO-based Small Cell Reassignments
}

\author{
Danny Finn*, Hamed Ahmadi*, Rouzbeh Razavi ${ }^{\dagger}$, Holger Claussen ${ }^{\dagger}$ and Luiz DaSilva* \\ ${ }^{*}$ CONNECT, Trinity College Dublin, Ireland \\ ${ }^{\dagger}$ Bell Laboratories, Alcatel-Lucent, Dublin, Ireland
}

\begin{abstract}
In this work we investigate the reassignment of User Equipments (UEs) between adjacent small cells to concurrently enable spatial multiplexing gains through Multi-User MIMO (MU-MIMO) and reductions in energy consumption though switching emptied small cells to a sleep state. We consider a case where UEs can be reassigned between adjacent small cells provided that the targeted neighbouring cell contains a UE with which the reassigned UE can perform MU-MIMO without experiencing excessive multi-user interference, and whilst achieving a minimum expected gain in spectral efficiency over the previous original cell transmissions as a result. We formulate the selection decision of which UEs to reassign as a set covering problem with the objective of maximising the number of small cell base stations to switch to a sleep state. Our results show that, for both indoor and outdoor LTE small cell scenarios, the proposed MU-MIMO-based reassignments achieve significant reductions in the required number of active small cell base stations, whilst simultaneously achieving increases in spectral efficiency.
\end{abstract}

Keywords-MU-MIMO, energy efficiency, small cells, sleep modes, inter-cell coordination, LTE.

\section{INTRODUCTION}

By significantly improving the spatial reusability of spectrum resources, small cells have shown huge potential to address the capacity deficit that mobile operators are expected to face in the near future. The reduced coverage range of small cell evolved Node Base stations (eNBs) implies that effective spatial reuse may require dense deployment of such cells, which leads to new radio resource management challenges.

MU-MIMO is a spatial multiplexing technique in which multiple transmit antennas at the eNB are used to simultaneously serve multiple UEs within a single time-frequency resource; this is achieved by transmitting to each UE on a different spatial layer. Essentially this consists of directing orthogonal beams at each served UE. Due to MU-MIMO's ability to provide large spatial multiplexing gains without requiring additional antennas on the UE, and its ability to overcome rank deficiency problems (which often limit single point-to-point spatial multiplexing), MU-MIMO capabilities have been highly emphasised in recent $3^{\text {rd }}$ Generation Partnership Program (3GPP) standardisation.

Often, however, MU-MIMO is limited to macrocell scenarios. The reason for this is that, in order to keep the interference between simultaneously served UEs (Multi-User Interference (MUI)) to a minimum, the UEs must experience sufficiently uncorrelated (semi-orthogonal) channels. In small cell scenarios, where there are few UEs per cell, it is not always possible to find suitable UE sets for MU-MIMO. However, in dense small cell scenarios a UE can often be in range of

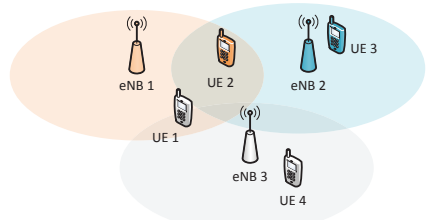

(a)

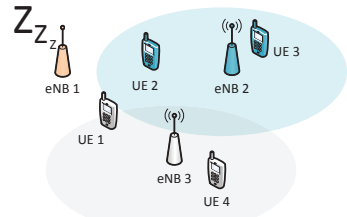

(b)
Fig. 1: UE colours indicate the Small Cell eNBs to which each UE is attached. (a) Consider that by reassigning UE 2 to eNB 2 MU-MIMO transmissions simultaneously serving UEs $2 \& 3$ can be enabled (e.g. if UE 2 is within range of eNB 2 and the channels of UEs $2 \& 3$ are semi-orthogonal). (b) Additionally, by reassigning UE 2 to eNB 2, as there are no more UEs attached to eNB 1 , eNB 1 can be switched to a sleep state to conserve energy.

multiple small cells. By reassigning UEs between neighbouring eNBs in such a way as to increase the number of suitable UE pairings, additional MU-MIMO spatial multiplexing gains can be enabled [1]. This can be seen as similar to enlarging the search space for suitable UE MU-MIMO pairings.

Given this scenario, once a UE is reassigned to a target neighbouring cell, if the host cell is no longer serving any active UEs, it can enter an idle state where radio transmissions are temporarily suspended, achieving high energy savings. An example of this is illustrated in Figure 11 This additionally results in a reduction in interference and pilot pollution problems, which are often observed in dense deployments. Moreover, considering that electricity costs account for 20-30 percent of network operational expenses [2], there is no lack of motivation for operators to become more energy efficient.

Within this context, this paper introduces a novel scheme where UEs are selected for reassignment to neighbouring eNBs in order to save energy by reducing the number of active small cell eNBs serving UEs, while simultaneously achieving spectral efficiency gains by enabling use of additional spatial layers through MU-MIMO. We perform system level simulations to quantify the achievable gains in two scenarios: one indoor and one outdoor.

\section{RELATED WORK}

In our previous work [1] we proposed a mechanism called MU-MIMO Across Small Cells in which UEs are reassigned between neighbouring eNBs to achieve an increase in spectral efficiency. The current work combines this concept with the use of small cell sleep modes to obtain simultaneous increases in both spectral and energy efficiency by reassigning UEs in such a way as to concurrently improve MU-MIMO operation and produce unoccupied small cells, which can then be switched to an idle state to conserve energy. To reflect this change of emphasis significant changes were required within the reassignment mechanism including modification of 
the method of selection of UEs to reassign (which is now formulated as a set covering optimisation), introduction of a new parameter $\tau$ to grant control over the tradeoff between energy and spectral efficiency, and considerations concerning sleep state operation.

As discussed in [2], sleep mode techniques often fall into one of three categories, differentiated by the method used for their reactivation. These are: Small Cell Controlled, in which an RF sniffer is utilised to identify potential UEs to serve [3]; Core Network Controlled, in which, as in our work, small cells are put into sleep states and re-awoken by a centralised core network element; and finally UE controlled, in which UEs emit periodic wake-up signals for surrounding small cells.

Traditionally these works have taken advantage of low traffic conditions and the energy saving possibilities they present, although more recently, given the density of small cell deployments and the associated cell redundancies/over provisioning, the work has evolved to also include user association considerations [4], [5]. In [4] centralised decisions are made to switch a portion of the active small cell eNBs to sleep modes, from which the resulting gains in energy efficiency and Signal to Interference and Noise Ratio (SINR) (as a result of reductions in inter-cell interference) are studied through system level simulation. Our work further improves the UE performance by selectively deactivating small cell eNBs in such a way that achieves both MU-MIMO spatial multiplexing and interference reduction spectral efficiency gains. In [5] uplink Cloud Radio Access Network (C-RAN) is considered with centralised joint decoding of the received signals of multiple multi-antenna small cell eNBs. The authors introduce a sparsity-inducing term (eNB activity cost) to their optimisation to jointly maximise rate and minimise the number of active small cells. Unlike [5] we do not consider joint encoding or decoding. Instead MU-MIMO transmissions are performed separately by each small cell eNB, which considerably reduces computation and coordination overheads.

The concept of coordinating the reassignment of UEs in order to achieve MU-MIMO gains can be related to a number of Coordinated Multi-Point (CoMP) concepts, most notably Coordinated Beamforming/Scheduling, Network MIMO and Dynamic Cell/Point selection. Our work differs considerably from these by actively reassigning UEs, avoiding joint transmissions and applying MU-MIMO considerations, respectively, as well as achieving additional gains through use of sleep states. [6] provides a recent survey on these and other similar multi-cell scheduling concepts.

\section{SYSTEM MODEL}

Our network contains $N_{U E}$ UEs, each with $N_{r}$ receive antennas, and $N_{e N B}$ eNBs, each with $N_{t}$ transmit antennas. The eNBs constitute a heterogeneous mix of tri-sector macrocell eNBs and open subscriber group small cell eNBs all operating within the same frequency band. Our UEs of interest are those served by the small cell eNBs and as such the macrocell eNBs are simply modelled as sources of inter-cell interference.

The small cell eNBs are governed by a central coordinator which is responsible for initiating reassignments and small cell sleep states. UEs are only served by a single eNB at any point in time. This means coherent transmissions from multiple cells are not necessary and the coordination requirements on the central controller are low relative to other CoMP techniques.

A small cell eNB $e$ can operate in either an active or idle state; indicated by $a_{e}=1$ or $a_{e}=0$, respectively.

\section{A. Signal Model}

For MU-MIMO with two UEs co-scheduled, each transmitting on a single spatial layer (rank-1), the received signal of UE $k$, co-scheduled for MU-MIMO with UE $j$, is given by

$$
\mathbf{y}_{k}=\mathbf{H}_{k, O} \mathbf{w}_{k} x_{k}+\mathbf{H}_{k, O} \mathbf{w}_{j} x_{j}+\sum_{l=1}^{N_{e N B}} a_{l} \mathbf{H}_{k, l} \mathbf{W}_{l} \mathbf{x}_{l}+\mathbf{n}_{\mathrm{W}, k}
$$

where $\mathbf{y}_{k}$ is the $N_{r} \times 1$ received signal vector, $\mathbf{H}_{k, O}$ is the $N_{r} \times N_{t}$ channel matrix within the original serving cell, $\mathbf{w}_{k}$ is the $N_{t} \times 1$ applied unitary precoding and $x_{k}$ is the transmitted symbol, of UE $k . \mathbf{H}_{k, O} \mathbf{w}_{j} x_{j}$ is the interference from the co-scheduled UE $j$, while $\mathbf{H}_{k, l} \mathbf{W}_{l} \mathbf{x}_{l}$ is the interference from neighbouring cell $l$ and finally $\mathbf{n}_{\mathrm{W}, k}$ is complex Additive White Gaussian Noise (AWGN), the elements of which have zero mean and variance $\sigma^{2}$. It should be noted that the value $\mathbf{H}_{k, O}$ in this equation includes the transmit power to each of the two co-scheduled UEs, which is one half of the power they would have if they were scheduled alone for Single-User MIMO beamforming on a single spatial stream (SU-MIMO). As indicated by $a_{l}$, if a neighbouring eNB is not active it does not produce intercell interference. It is assumed that all serving cells are active, hence we omit the $a_{O}$ which would otherwise precede $\mathbf{H}_{k, O}$.

The post-reception SINR of UE $k$, after the application of a $1 \times N_{r}$ receive filter $\mathbf{g}_{k}$, can be represented as

$$
\gamma_{k}=\frac{\left|\mathbf{g}_{k} \mathbf{H}_{k, O} \mathbf{w}_{k}\right|^{2}}{\left|\mathbf{g}_{k} \mathbf{H}_{k, O} \mathbf{w}_{j}\right|^{2}+\left|\mathbf{g}_{k} \sum_{l=1}^{N_{e N B}-1} a_{l} \mathbf{H}_{k, l} \mathbf{W}_{l}\right|^{2}+\left.\sigma^{2} \mathbf{I}|| \mathbf{g}_{k}\right|^{2}}
$$

We apply a Minimum Mean Square Error (MMSE) with Interference Rejection Combining (IRC) filter [7]. This filter combines the suppression of co-layer intra-cell interference by the MMSE filter and suppression of inter-cell interference through IRC filtering. This receiver is also known as the Advanced Long Term Evolution (LTE) UE Receiver and is the baseline receiver from 3GPP LTE Rel.11 onward [8].

\section{B. CSI calculations}

The LTE standards specify three types of Channel State Information (CSI) feedback for use in the scheduling of UEs and adaptive modulation and coding. The Channel Quality indicator (CQI) is a quantised form of SINR, the Precoding Matrix Indicator (PMI) recommends a linear precoding matrix from a predefined codebook, and the Rank Indicator (RI) indicates the rank (number of parallel transmission streams) to use [9]. For both SU-MIMO and MU-MIMO operation, we consider only Rank-1 transmissions for each UE.

In this work, we consider wideband PMI feedback. The PMI is selected as the one which maximises the SU-MIMO mutual information summed over all subcarriers in the channel bandwidth. We consider LTE Rel.10 MU-MIMO operation which supports the subband use of MU-MIMO on some subcarriers and SU-MIMO on others, as well as supporting the use of Zero-forcing to orthogonalise non-orthogonal precoders in Semi-orthogonal User Selection (SUS). For 2 or 4 Transmit (Tx) antenna configurations Rel.10 MU-MIMO makes use of the Rel.8 MU-MIMO precoding codebook. 
1) MU-MIMO CQI calculation: Computing CQI feedback for MU-MIMO scenarios is always a problem as the MultiUser Interference (MUI) is a function of which UEs are selected to be simultaneously served. Furthermore, if the channel conditions do not suit MU-MIMO the scheduler may fallback to SU-MIMO operation. This makes it not possible to characterise the SINR prior to the scheduling process. For this reason, we model the MU-MIMO CQI as proposed in [10]. All UEs feed back CQIs for SU-MIMO (not including MUI) which are adjusted to account for the splitting of the transmit power between the co-scheduled UEs, and for the mean level of unsuppressed MUI, $\Delta_{M U I}$, as follows [10]:

$$
C Q I_{\mathrm{MU}-\mathrm{MIMO}}=\frac{1}{\frac{n_{M U}}{C Q I_{\mathrm{SU}-\mathrm{MIMO}}}+\Delta_{M U I}\left(n_{M U}-1\right)}
$$

where $n_{M U}$ is the number of UEs co-scheduled for MU-MIMO within a single time-frequency Resource Block (RB) and $C Q I_{\text {SU-MIMO }}$ is the fed back quantised SINR in linear form.

$\Delta_{M U I}$ is a precomputed parameter, specific to the environment and device configuration. It is obtained by taking the expectation over a large number of channel realisations of the ratio of the suppressed MUI power to the SU-MIMO signal power (without power splitting between co-scheduled UEs):

$$
\Delta_{M U I}=\mathbb{E}\left[\frac{\left|\mathbf{g}_{k} \mathbf{H}_{k, O} \mathbf{w}_{j}\right|^{2}}{n_{M U}\left|\mathbf{g}_{k} \mathbf{H}_{k, O} \mathbf{w}_{k}\right|^{2}}\right] .
$$

A fixed value of $\Delta_{M U I}$ is made reasonable by the constraint that the maximum allowed channel correlation of simultaneously served UEs, $\epsilon$, is very low. This means that between different effective channel matrix realisation pairs the variance in the amount of suppression that can be achieved is also low.

2) Neighbouring eNB Feedback: In order to assess the feasibility of potential reassignments, when the reassignment mechanism is initiated the UE needs to check the quality of the channels between it and any target neighbouring eNBs. To this end, as detailed later in Section IV, unpaired UEs compute a wideband PMI and a wideband CQI (CQI averaged across subbands over the transmission bandwidth using Mutual Information Effective SINR Mapping (MIESM), or similar) for the target cell.

In LTE systems the Cell Specific Reference Signal (CS-RS) offers the primary means of neighbouring cell CSI estimation. CS-RS locations of neighbouring cells are shifted in the frequency-domain based on a $\bmod (6)$ of their cell ID [11]. This was originally intended to provide frequency-domain orthogonality between the reference signals of neighbouring cells in a macrocell hexagonal grid structure, although the CS-RSs are also coded with cell-specific Zadoff-Chu sequences to provide orthogonality in denser deployment cases (e.g. small cell scenarios). CQI values obtained in this way are then adapted for different values of $n_{M U}$ according to Eqn. (3).

In this work, the target cell CQI is used only in the reassignment decision, and reassignments are only performed which enable the deactivation of the original cell. Hence, we do not include interference from the original eNB in the target cell CQI. This provides a more accurate estimate of the postreassignment rate. Nonetheless, it is not possible to know, prior to the reassignment decision, which other neighbouring eNBs will also be deactivated. If additional neighbouring eNBs are deactivated the levels of interference will be further reduced, thus the target cell CQI provides a conservative estimate of the post-reassignment rate.

\section{MU-MIMO Spectral Efficiency}

In both the scheduling and reassignment processes it is necessary to compare the expected relative performances of SU-MIMO and MU-MIMO. For this, we map the $C Q I_{\text {SU-MIMO }}$ and $C Q I_{\text {MU-MIMO }}$ values to expected rates per $\mathrm{RB}$ based on a fitting to Bit-Interleaved Coded modulation (BICM) curves, as discussed in [12]. We represent the expected rate of a UE $k$, when co-scheduled as one of $n_{M U}$ UEs, attached to eNB $e$ as $r_{n_{M U}, k, e}$. In this work $e$ will be either the currently attached eNB, which we will call the original eNB, $O$, or a neighbouring eNB targeted for reassignment, $T$.

When MU-MIMO is used $\left(n_{M U} \geq 2\right)$, a single $\mathrm{RB}$ is shared amongst multiple co-scheduled UEs, each on different spatial layers; as the UEs each only part-occupy the RB bandwidth, their MU-MIMO spectral efficiency in bits per RB is given by their rate for the $\mathrm{RB}$, multiplied by the number of simultaneously served UE that the RB is divided between.

\section{Energy Consumption Model}

We model eNB input power consumption according to the framework set out by the FP7 EARTH Project [13] as follows:

$$
P_{\text {in }}=\left\{\begin{array}{cc}
N_{t}\left(P_{0}+\Delta_{P} P_{\text {out }}\right), & \text { ACTIVE } \\
N_{t} P_{\text {sleep }}, & \text { IDLE }
\end{array}\right.
$$

where $P_{0}$ represents the power consumption at zero RF output power, $P_{\text {out }}$ represents the load-dependent RF output power which has a maximum of $P_{\max }$ and slope $\Delta_{P}$. For full buffer traffic, as in this work, transmissions will operate at maximum power, meaning that $P_{\text {out }}$ for active eNBs will be $P_{\text {max }} . P_{\text {sleep }}$ represents the power consumed by an eNB in sleep mode. An eNB can switch into a sleep mode to save energy if there are no UEs requesting transmissions which it has to serve. These power consumption values will differ depending on the type of base station considered, be it femto, pico or macro.

\section{REASSIGNMENT MECHANISM}

The MU-MIMO across Small Cells reassignment mechanism of this work follows a common structure to our previous work [1], augmented by the deactivation of idle small cells, and consists of five main steps:
A. Selection of Considered UEs
B. Check for Target UEs
C. Selection of UEs to reassign from Reassignable UEs
D. Reassignment
E. Deactivation of emptied small cells.

Additionally, as necessitated by the energy consumption minimisation objective of the reassignment, the third step, in which UEs are selected for reassignment, is also added. 


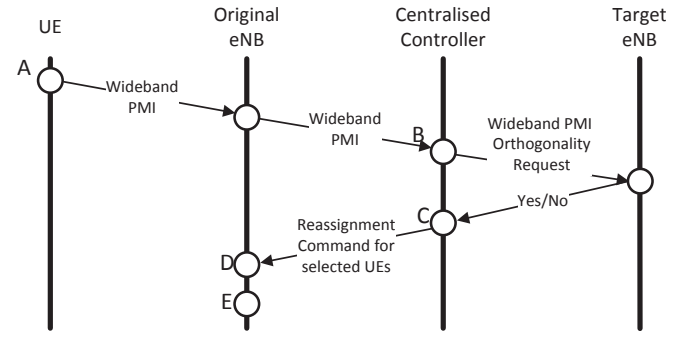

Fig. 2: MU-MIMO Across Small Cells with sleep mode usage. A. UEs assess whether they should be considered for reassignment. B. The Centralised Controller checks the target eNBs for potential Target UEs with which the Considered UEs can be co-scheduled for MU-MIMO. If such Target UEs exist, the corresponding considered UEs are termed reassignable. C. The Centralised Controller selects a subset of the Reassignable UEs to reassign. In this paper, these are selected so as to minimise the required number of active eNBs. D. The selected UEs get reassigned. E. eNBs with no remaining attached UEs switch to a sleep state to conserve energy.

\section{A. Selection of Considered UEs}

To avoid unnecessary exchange of feedback information, at the start of the reassignment process, UEs assess whether they should be considered for MU-MIMO-based cell reassignment.

A UE capable of MU-MIMO operation in its current cell is unlikely to improve its MU-MIMO capabilities by reassigning to a neighbouring cell and is thus removed from the set of Considered UEs. These removed UEs meet the constraint

$$
2 \overline{r_{2, k, O}} I_{k, O}>\overline{r_{1, k, O}} \text {. }
$$

where $\overline{r_{n, k, e}}$ denotes the expected instantaneous rate averaged over all RBs. $n=1$ and $n=2$ denote SU- and two-layer MU- MIMO, respectively, and the averaged rates are scaled by the number of co-scheduled UEs (layers) to correspond to the average spectral efficiencies in bits per RB. $I_{k, e}$ indicates the presence of another UE attached to eNB $e$ with a precoding matrix semi-orthogonal to that of UE $k$.

Of these UEs, those which expect a predefined minimum gain from MU-MIMO usage in a neighbouring eNB are considered for reassignment:

$$
2 \overline{r_{2, k, T}}-\overline{r_{1, k, O}}>\tau .
$$

where $\tau$ is an offset indicating how much higher the target cell expected rate must be, which can be either positive or negative, and $\overline{r_{2, k, T}}$ is obtained from neighbouring eNB reference signals as outlined in III-B2

The parameter $\tau$ is introduced to provide control over the tradeoff between spectral and energy efficiency. A positive value of $\tau$ indicates a strict requirement on the minimum expected spectral efficiency gains, while a negative value of $\tau$ indicates the willingness to sacrifice some spectral efficiency for a reduction in energy consumption.

UEs meeting constraints (6) and (7) are then labelled the Considered UEs. These Considered UEs share their wideband PMI, for each target neighbouring eNB, with the central coordinator via their currently attached eNB.

\section{B. Check for Target UEs}

We term Target UEs as UEs attached to the target neighbouring eNB with which the Considered UE could potentially be co-scheduled for MU-MIMO. The central controller checks for Target UEs by checking if the precoder of any UE in the neighbouring cell is semi-orthogonal to that of the considered UE (corresponding to the fed back wideband PMI), and that their SINR is not too low for MU-MIMO operation to be beneficial. Any Considered UEs for which a Target UE exists are termed Reassignable UEs.

\section{Selection of UE to reassign from the Reassignable UEs}

The choice of UEs to reassign can determine the number of eNBs which can be deactivated as a result of the reassignment, as well as the increases in spectral efficiency and MU-MIMO usage that can be achieved.

In this work we formulate this decision as a set covering problem in which we select UEs to reassign so as to maximise the number of deactivated eNBs. In this, the set of all UEs $k \in \mathcal{U}$ must be covered (served) by the set of active base stations. However a UE $k$ can only be served by an eNB $e \in \mathcal{E}$ if either $e$ is $k$ 's original eNB or $k$ can be reassigned to $e$.

This set covering problem can be expressed as follows:

$$
\begin{array}{lc}
\text { minimize } & \sum_{e \in \mathcal{E}}\left(a_{e}\right) \\
\text { subject to } & \sum_{e \in f(k)}\left(a_{e}\right) \geq 1 \quad \forall k \in \mathcal{U} \\
a_{e} \in\{0,1\} \quad \forall e \in \mathcal{E}
\end{array}
$$

where $a_{e}$ indicates that eNB $e$ is active and $f(k)$ indicates the set of eNBs which can serve UE $k$.

In order to assess the performance of the mechanism under optimal reassignment selection (maximum number of deactivated eNBs) we solve this problem using the IBM ILOG CPLEX Optimiser. The set covering problem is NP-hard; however, as our problem size is relatively small, CPLEX finds solutions in a reasonable amount of time. Nonetheless for real implementations a heuristic solution would be required, for which polynomial time heuristics achieving $\Theta\left(\ln N_{U E}\right)$ approximations exist [14].

\section{Reassignment}

All UEs attached to the small cell eNBs selected to switch to sleep mode are reassigned to their respective target eNBs.

\section{E. Deactivation of emptied small cells}

Once these UEs have been reassigned, the emptied small cell eNB can enter the idle mode regime to minimise energy consumption and to avoid creating unwanted interference. However, there is a need for effective mechanisms that can detect the presence of a new UE and subsequently wake up the base station. This can be done in either a distributed or centralised fashion.

The simplest distributed wake up mechanism is to equip the eNB with an RF sniffer which monitors detected energy in the uplink band. Once this quantity exceeds a certain threshold, the eNB can interpret this as a sign indicating the presence of a nearby UE [3]. However, setting the detection threshold optimally is difficult and, contrary to the scenario in [3] where the UE communicates with an underlying macrocell $\mathrm{eNB}$, in dense deployment scenarios, the UE is more likely to be served by a neighbouring small cell eNB. This implies that the UE transmits at lower power, making detection more difficult. 


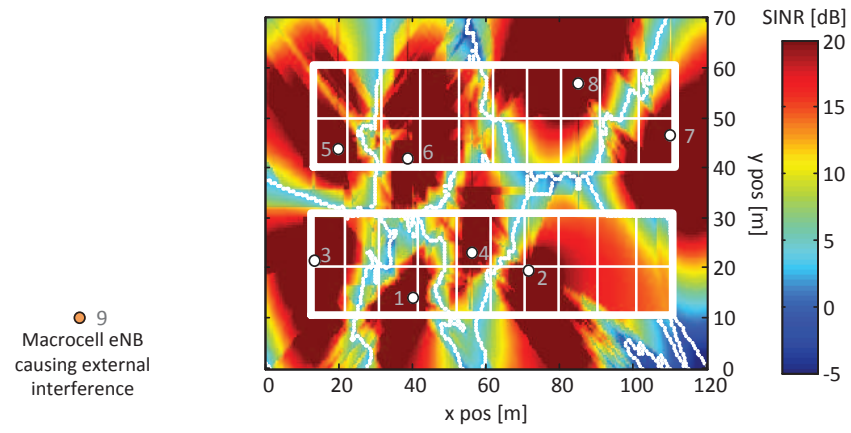

Fig. 3: Dual Stripe scenario SINR distribution from simulation with a DR of 0.2 . White dots mark the HeNB locations, the orange dot marks the location of the external macrocell.

In the centralised approach a separate network element triggers the small cell eNB to wake up. This has the advantage that the base station will not be triggered unnecessarily if the UE can be served by other existing eNBs. The challenging task is to determine when a UE falls under the coverage area of a given small cell base station. This can be done using RF-fingerprints to estimate the UE's location or through other advanced geolocation methods.

As our network already contains a centralised controller the centralised approach presents the best fit to our scenario. Furthermore, this approach provides a higher energy efficiency gain than the distributed approach, as less infrastructure is required to detect when a reactivation is required [2].

\section{InVESTIGATED SCEnARIOS AND SETTINGS}

We explore the potential benefits of the proposed coordinated MU-MIMO-based reassignment and sleep mode activation mechanism in two small cell scenarios, both subject to external macrocell interference. The first is an indoor residential femtocell deployment, while the second is an outdoor picocell deployment in the commercial centre of a major European city.

\section{A. Indoor Residential Scenario}

For indoor residential femtocell (HeNB) deployments the 3GPP Dual Stripe model [15] is the most widely used. This model consists of two apartment buildings side-by-side, each subdivided into apartments separated by walls. The density of HeNBs deployed is indicated by the Deployment Ratio (DR), which denotes the probability of a given apartment containing a HeNB. We consider a single-story deployment with a DR of 0.2 (8 HeNBs deployed on average). The SINR distribution of an example deployment is illustrated in Figure 3.

\section{B. Outdoor City Centre Scenario}

We also study the performance of our proposed mechanism in an outdoor scenario, the area surrounding Stachus Square, a commercial zone in the city centre of Munich, Germany. Figure 4 shows the maximum picocell Reference Signal Received Power (RSRP) within the $250 \mathrm{~m}$ x $250 \mathrm{~m}$ investigated region. The detailed 3D ray-tracing tool Wireless System Engineering (WiSE) [16] was used to accurately compute the pathloss and shadow fading in this scenario taking fully into account the locations of the eNBs, building and other obstacles.

\section{Common Parameters/ General Settings}

All eNBs each have four closely spaced transmit antennas, while the UEs have two receive antennas which use MMSEIRC. At the start of simulation all small cell eNBs operate in

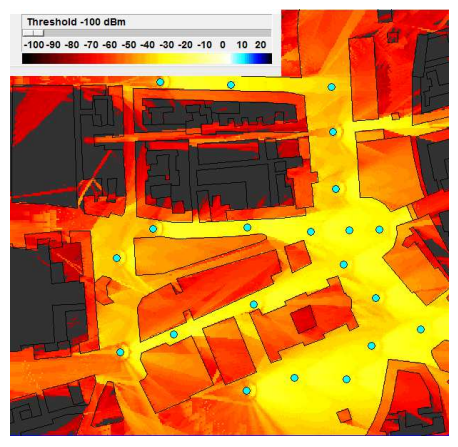

Fig. 4: Maximum RSRP map for Munich picocell deployment. The locations of the 21 picocell eNBs are marked by cyan dots. UEs also experience macrocell interference from 6 surrounding tri-sector base stations the locations of which correspond to those of one of Germany's top-tier mobile operators.

an active state and have the same number of allocated UEs. UEs are initially assigned to the eNB with the highest RSRP. UEs can only be reassigned between small cells and cannot be reassigned to a macrocell.

Both before and after the reassignments, UEs are scheduled using Semi-orthogonal User Selection (SUS) [17] modified for proportional fairness. This algorithm first selects the UE with the highest proportional fair metric to be scheduled, where the proportional fair metric is the ratio of the instantaneous achievable rate to the long-term average throughput of a UE. Next a set is computed of the UEs with quantised channel vectors semi-orthogonal to that of the the first selected UE 1 From this semi-orthogonal set the UE with the highest proportional fair metric is selected to be paired with the already scheduled UE. Finally, a check is performed to ensure that the proportional fair metric of the two selected UEs both performing MU-MIMO exceeds that of the first UE alone performing SU-MIMO. Whichever of the two possibilities has the better performance is then used. To avoid excessive user specific reference signalling overhead and as there are few UEs per cell, the maximum number of UEs that can be spatially coscheduled for MU-MIMO in a single RB is two; however, to support $n>2$, the mechanism would not require much change.

The parameters used in the energy efficiency model are those provided in [13] adjusted for the 4 transmit antenna case such that the maximum radiated power is kept consistent. Additional simulation parameters are provided in Table []

\section{RESULTS}

The following simulations were performed using the Matlab-based Vienna LTE System-Level simulator [19]. To enable this investigation, we implemented as extensions MU-MIMO scheduling and transmissions with MMSE-IRC receivers, the investigated indoor and outdoor deployment scenarios, and small cell energy expenditure computation.

Monte Carlo simulations were performed as a series of snapshots which were averaged over a large number of iterations to generate the presented results. Once per snapshot the MU-MIMO across Small Cells reassignment mechanism, described in section IV is carried out. We assess what energy

\footnotetext{
${ }^{1}$ The quantised channel vectors of a UE is obtained from the fed back PMI as the pseudo-inverse of the recommended precoding vector, and two quantised channel vectors are called semi-orthgonal if the spatial correlation between them is below a predefined bound, $\epsilon$.
} 
TABLE I: Simulation parameters

\begin{tabular}{|c|c|c|}
\hline Scenario Specific Parameters & 3GPP Dual Stripe (Indoor) & Munich (Outdoor) \\
\hline Pathloss Model & 3GPP Dual Stripe 15 & WiSE $[16$ \\
\hline Fast Fading Model & Winner II 18 & Winner II $[18]$ \\
\hline Deployment Densities & $\mathrm{DR}=0.2$ & Mean inter-site distance $=37 \mathrm{~m}$ \\
\hline Number of Small cells & 8 on average & 21 \\
\hline Small cell maximum Tx Power & $20 \mathrm{dBm}$ & $24 \mathrm{dBm}$ \\
\hline Number of Macrocell interferers & 3 & 18 \\
\hline Macrocell maximum Tx Power & $46 \mathrm{dBm}$ & $43 \mathrm{dBm}$ \\
\hline$P_{\max }$ & $0.025 \mathrm{~W}$ & $0.065 \mathrm{~W}$ \\
\hline$P_{0}$ & $2.4 \mathrm{~W}$ & $3.4 \mathrm{~W}$ \\
\hline$\Delta_{P}$ & 4.0 & 8.0 \\
\hline$P_{\text {sleep }}$ & $1.45 \mathrm{~W}$ & $2.15 \mathrm{~W}$ \\
\hline & \multicolumn{2}{|c|}{ General Parameters } \\
\hline Bandwidth & \multicolumn{2}{|c|}{$10 \mathrm{MHz}$} \\
\hline Snapshot length & \multicolumn{2}{|c|}{$\begin{array}{c}10 \text { TTIs } \\
\text { (5 TTIs before and } 5 \text { TTIs after reassignment) }\end{array}$} \\
\hline Channel feedback delay & \multicolumn{2}{|c|}{$1 \mathrm{~ms}$} \\
\hline eNB Antenna Configuration & \multicolumn{2}{|c|}{$\begin{array}{c}4 \mathrm{Tx} \text { antennas } \\
\text { Cross-polarised } 0.5 \lambda \text { spacing, }-45^{\circ} /, 45^{\circ} \text { slants }\end{array}$} \\
\hline UE Antenna Configuration & \multicolumn{2}{|c|}{$\begin{array}{c}\text { 2 Receive (Rx) antennas } \\
\text { Cross-polarised } 0.5 \lambda \text { spacing, } 0^{\circ} /, 90^{\circ} \text { slants }\end{array}$} \\
\hline MIMO transmission scheme & \multicolumn{2}{|c|}{$\begin{array}{c}\text { SU-MIMO: single layer } \\
\text { MU-MIMO: max. } 2 \text { UEs, } 1 \text { layer per UE }\end{array}$} \\
\hline Precoding Codebook & \multicolumn{2}{|c|}{ Rel.8 4 Tx codebook } \\
\hline Initial Cell Selection & \multicolumn{2}{|c|}{ Maximum RSRP } \\
\hline Feedback (To assigned cell) & \multicolumn{2}{|c|}{ Subband CQI, wideband PMI for all UEs } \\
\hline Feedback (To centralised controller) & \multicolumn{2}{|c|}{ Wideband PMI for each Target Neighbouring eNB } \\
\hline MU-MIMO $\Delta_{M U I}$ & \multicolumn{2}{|c|}{0.05} \\
\hline UE Scheduling & \multicolumn{2}{|c|}{ Proportional Fair SUS } \\
\hline SUS const $\epsilon$ & \multicolumn{2}{|c|}{0.1} \\
\hline Traffic Model & \multicolumn{2}{|c|}{$\begin{array}{c}\text { Full Buffer } \\
4 \text { Tx SU-MIMO with random PMI }\end{array}$} \\
\hline Inter-cell Interference model & \multicolumn{2}{|c|}{4 Tx SU-MIMO with random PMI } \\
\hline Feedback Overhead & \multicolumn{2}{|c|}{$31.15 \%$} \\
\hline
\end{tabular}

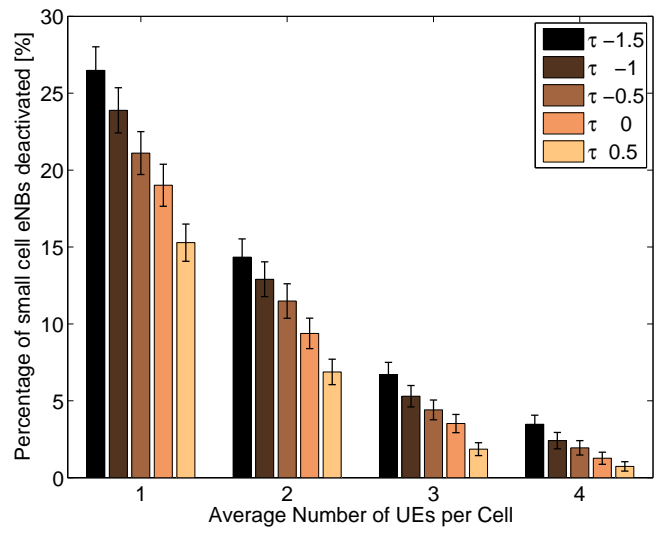

Fig. 5: Percentage of eNBs that can be switched to IDLE as a result of MU-MIMO-based cell reassignments for different reassignment thresholds $\tau$ in the indoor Dual Stripe scenario with DR of 0.2.

saving and spectral efficiency gains are achievable relative to the initial RSRP-based assignments, which represent current practice in small cell deployments. We investigate how how these are affected by the number of UEs per cell, the reassignment parameter $\tau$, and the reassignment selection algorithm. Vertical bars in all figures in this section correspond to the 95\% confidence interval.

\section{A. Energy Efficiency Gains}

Figures 5 and 6 show the percentage of small cell eNBs which are switched to a sleep mode as a result of the reassignment mechanism for the indoor Dual Stripe and outdoor Munich scenarios, respectively. As can be seen in both figures the percentage of small cell eNBs switched off decreases as the reassignment metric threshold $\tau$ increases. Higher $\tau$ corresponds to a stricter constraint on the foreseen reassignment spectral efficiency gains while increased leniency in $\tau$ results in a larger set of reassignable UEs, allowing more small cell eNBs to be switched off. While not shown, as $\tau$ continues to decrease, a point will be reached (roughly $\tau=-6$ ) where UE

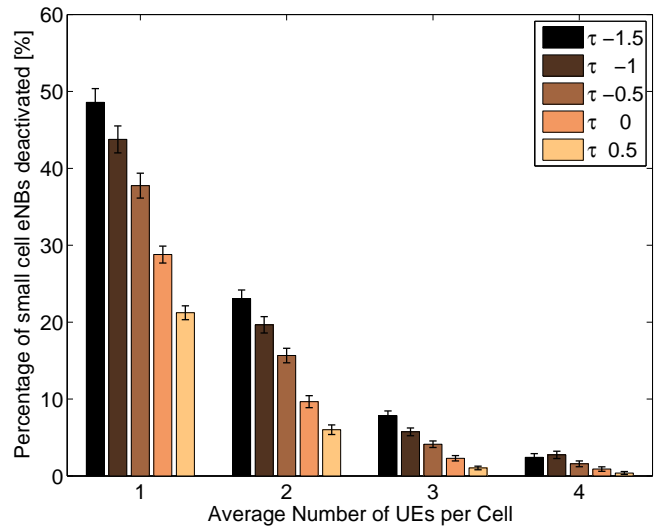

Fig. 6: Percentage of eNBs that can be switched to IDLE as a result of MU-MIMO-based cell reassignments for different reassignment thresholds $\tau$ in the outdoor Munich scenario.

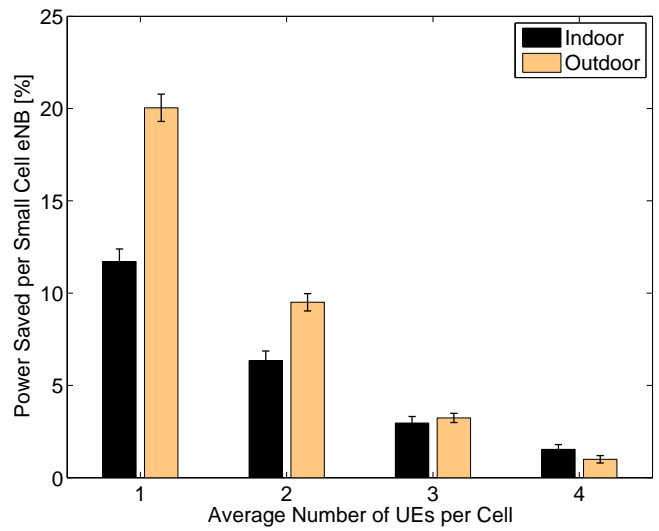

Fig. 7: Average saved eNB power consumption through switching eNBs to IDLE state as a result of MU-MIMO-based cell reassignments. $\tau=-1.5$.

reassignability becomes exclusively a function of UE channel orthogonality and no longer of the neighbouring cell SINRs. In this case a UE may be reassigned to any neighbouring eNB regardless of how far apart they may be, potentially resulting in dramatic decreases in spectral efficiency and inability to use MU-MIMO in the target neighbouring cell. Instead, in the figures we show a reasonable range of $\tau$ values for which this does not occur.

The more UEs there are per eNB the less likely all UEs in the cell will be reassignable. Further, if there are more UEs in the original cell it is more likely that suitable MU-MIMO pairs will already exist, reducing the number of considered UEs. This results in very low probabilities of a reassignment occurring for 4 UEs per cell, even for low $\tau$.

Comparing Figures 5 and 6 , the proportion of eNBs deactivated is generally higher in the outdoor scenario. Figure 7 presents the savings in small cell power consumption for the case of $\tau=-1.5$. We see that the power savings in the outdoor scenario are higher for few UEs per cell, while when there are many UEs per cell the power savings are comparable.

We see that in the outdoor case with 1 UE per cell a $15 \%$ reduction in the consumed power can be achieved. This corresponds to a $45 \%$ energy saving on the $37 \%$ percent the pico eNBs which got deactivated or an average saving of $4.5 \mathrm{~W}$ per pico cell. In the indoor case with the same parameters on average $2.5 \mathrm{~W}$ per femto cell can be saved. 


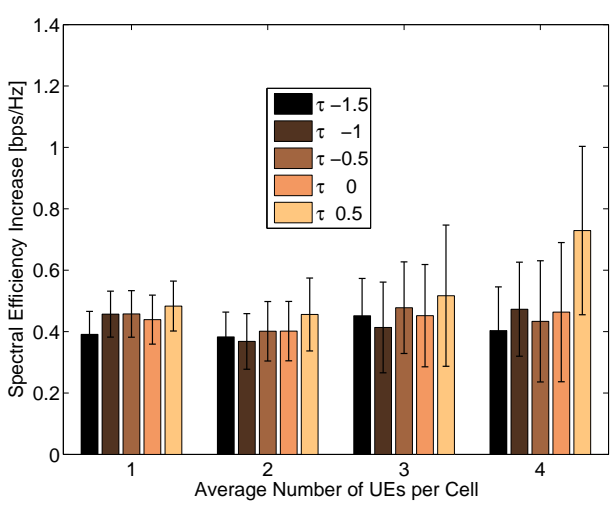

Fig. 8: Increases in Spectral Efficiency resulting from reassignment, averaged across the Reassigned and Target UEs in the Dual Stripe indoor scenario.

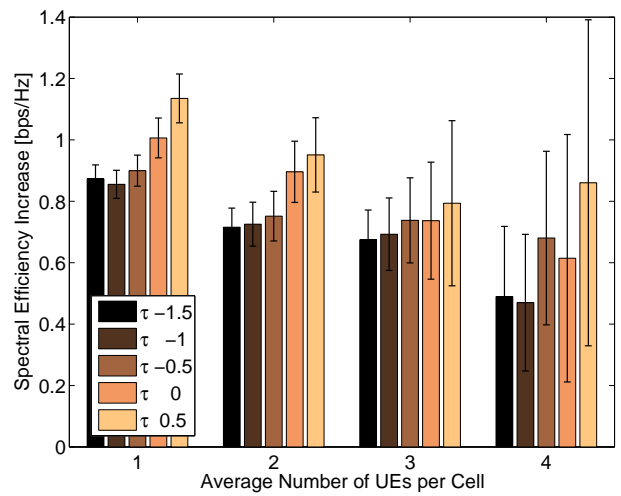

Fig. 9: Increases in Spectral Efficiency resulting from reassignment, averaged across the Reassigned and Target UEs in the Munich outdoor scenario.

\section{B. Spectral Efficiency Gains}

Figures 8 and 9 show the gains in spectral efficiency averaged over the UE that gets reassigned and the Target UE with which the Reassigned UE is simultaneously served for MU-MIMO in the target neighbouring cell. Equivalently, these are the gains in spectral efficiency of the enabled MU-MIMO usage over the pre-reassignment Single-User MIMO (SU-MIMO) usage, and are a function of the original and target eNB SINRs, before and after reassignment.

As expected, higher reassignment threshold $\tau$ generally corresponds to higher spectral efficiency increase; however, the effect of $\tau$ is not strong. Due to it not being possible to know prior to reassignment which neighbouring cells will also be deactivated, $\tau$ cannot take into account gains in SINR from a neighbouring eNB switching to sleep state (thus removing the inter-cell interference it causes). Further, lower values of $\tau$ mean that more reassignments can be performed, allowing more small cells to be deactivated, and resulting in more reductions in interference. This also explains why the increases in spectral efficiency tend to exceed the values of $\tau$.

We see that the gains in spectral efficiency in the outdoor scenario are higher than in the indoor scenario. This is due to the lack of walls between neighbouring small cell eNBs on the same street. In the indoor scenario there are walls between all eNBs, meaning that the minimum difference in SINR between two neighbouring cells is higher. This further explains why the proportion of eNBs that could be deactivated was higher in the outdoor scenario in Figure 6.

\section{CONCLUSions}

In this work we have demonstrated the combined use of MU-MIMO-based UE reassignments and centralised control of small cell sleep states to achieve simultaneous increases in spectral efficiency and reductions in energy consumption. We compare the achievable gains in two small cell scenarios, one indoor residential apartment block scenario (Dual Stripe) and one commercial city centre outdoor scenario (Stachus Square, Munich). We found that it was possible to switch over $25 \%$ (indoor) and 35\% (outdoor) of small cells to a sleep state whilst still achieving considerable gains in spectral efficiency. Based on the energy consumption model used [13] these correspond to power savings of $12 \%$ and $15 \%$ respectively.

\section{ACKNOWLEDGMENT}

We gratefully acknowledge Dr. Andrea F. Cattoni for his much appreciated input in this and our previous work.

This material is based upon works supported by the Science Foundation Ireland under Grants No. 10/CE/I1853 and 10/IN.1/I3007.

\section{REFERENCES}

[1] D. Finn, H. Ahmadi, A. Cattoni, and L. DaSilva, "Multi-User MIMO across Small Cells," in IEEE ICC, Jun. 2014.

[2] I. Ashraf, F. Boccardi, and L. Ho, "SLEEP mode techniques for small cell deployments," IEEE Comm.s Mag., vol. 49, no. 8, 2011.

[3] I. Ashraf, L. T. W. Ho, and H. Claussen, "Improving Energy Efficiency of Femtocell Base Stations Via User Activity Detection," in IEEE WCNC, 2010.

[4] Y. Li, Y. Jia, Y. Wang, and Q. Liu, "Collaborative Sleeping Scheme for Femtocell Networks," in IEEE GreenCom, iThings and CPSCom, 2013.

[5] S.-H. Park, O. Simeone, O. Sahin, and S. S. (Shitz), "Robust and Efficient Distributed Compression for Cloud Radio Access Networks," IEEE Transactions on Vehicular Technology, vol. 62, no. 2, 2013.

[6] E. Pateromichelakis, M. Shariat, A. ul Quddus, and R. Tafazolli, "On the Evolution of Multi-Cell Scheduling in 3GPP LTE / LTE-A," IEEE Communications Surveys \& Tutorials, vol. 15, no. 2, 2013.

[7] L. F. Del Carpio Vega, "System level modeling and evaluation of advanced linear interference aware receivers," M.Sc., School of Elec. Eng., Aalto Univ., Finland, 2012.

[8] H. Holma and A. Toskala, LTE-Advanced: 3GPP Solution for IMTAdvanced. Wiley, 2012.

[9] 3GPP TS 36.213, "Evolved Universal Terrestrial Radio Access (EUTRA); Physical layer procedures," Tech. Rep.

[10] H. T. Nguyen and I. Z. Kovacs, "A MU-MIMO CQI Estimation Method for MU-MIMO UEs in LTE Systems," IEEE VTC2012-Fall.

[11] 3GPP TS 36.211, "E-UTRA; Phy channels and modulation," Tech. Rep.

[12] S. Schwarz, C. Mehlfuhrer, and M. Rupp, "Low complexity approximate maximum throughput scheduling for LTE," in IEEE Signals, Systems and Computers (ASILOMAR), 2010, pp. 1563-1569.

[13] M. A. Imran et al., "FP7 EARTH D3.2: Energy efficiency analysis of the reference systems, areas of improvements and target breakdown," Tech. Rep., 2010.

[14] V. Chvatal, "A greedy heuristic for the set-covering problem," Mathematics of operations research, vol. 4, no. 3, pp. 233-235, 1979.

[15] 3GPP RAN 4, "Simulation assumptions and parameters for FDD HeNB RF requirements." R4-092042, 2009.

[16] S. Fortune et al., "WISE design of indoor wireless systems: practical computation and optimization," IEEE CS\&E, vol. 2, no. 1, 1995.

[17] T. Yoo, N. Jindal, and A. Goldsmith, "Multi-Antenna Downlink Channels with Limited Feedback and User Selection," IEEE JSAC, vol. 25, no. 7, 2007.

[18] P. Kyösti et al., "Matlab Implementation of the WINNER Phase II Channel Model ver1.1," Tech. Rep., 2007.

[19] J. C. Ikuno, M. Wrulich, and M. Rupp, "System Level Simulation of LTE Networks," in IEEE VTC2010-Spring. 